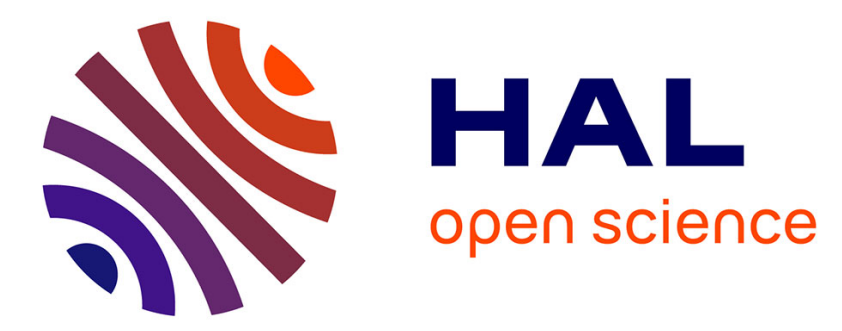

\title{
Linear magneto-viscoelastic model based on magnetic permeability components for anisotropic magnetorheological elastomers
}

\author{
I. Agirre-Olabide, P. Kuzhir, M.J. J Elejabarrieta
}

\section{- To cite this version:}

I. Agirre-Olabide, P. Kuzhir, M.J. J Elejabarrieta. Linear magneto-viscoelastic model based on magnetic permeability components for anisotropic magnetorheological elastomers. Journal of Magnetism and Magnetic Materials, 2018, 446, pp.155-161. 10.1016/j.jmmm.2017.09.017 . hal-01672508

\author{
HAL Id: hal-01672508 \\ https://hal.science/hal-01672508
}

Submitted on 25 Dec 2017

HAL is a multi-disciplinary open access archive for the deposit and dissemination of scientific research documents, whether they are published or not. The documents may come from teaching and research institutions in France or abroad, or from public or private research centers.
L'archive ouverte pluridisciplinaire HAL, est destinée au dépôt et à la diffusion de documents scientifiques de niveau recherche, publiés ou non, émanant des établissements d'enseignement et de recherche français ou étrangers, des laboratoires publics ou privés. 


\title{
On the magneto-viscoelastic modelling of anisotropic magnetorheological elastomers
}

\author{
I Agirre-Olabide ${ }^{1}$, P. Kuzhir ${ }^{2}$ and M J Elejabarrieta ${ }^{1}$ \\ ${ }^{1}$ Mech. \& Manuf. Dept., Mondragon Unibertsitatea, 20500 Arrasate-Mondragon, Spain \\ ${ }^{2}$ University Côte d'Azur, CNRS UMR 7010, Institute of Physics of Nice, Parc Valrose, 06100 Nice, France \\ E-mail: mjelejabarrieta@mondragon.edu
}

\begin{abstract}
The storage modulus variation of anisotropic magnetorheological elastomers (MREs) induced by an external magnetic field was modelled in the frequency domain. This involves synthesising five anisotropic MREs with different particle content and measuring its dynamic and magnetic properties. Dynamic properties were measured using a rheometer equipped with a magnetorheological cell and the magnetic permeability of each sample was measured with a vibrating sample magnetometer. Scanning Electron Microscope images were used to determine particle distribution. A four parameter fractional derivative model was used to describe MRE viscoelasticity in the absence of magnetic field and the fitting error was not larger than 1\%. Magneto-induced modulus was also studied and two different models were analysed, the one of Jolly et al. (Smart Mater Struct;5:607 (1999)) and the other one of López-López et al. (J Rheol. 56:1209 (2012)). The first model underestimated the influence of the magnetic field for low particle contents while at high ones it overestimated the magnetic field effect, up to $13 \%$. However, in the second model magnetic permeability values were used, and the error between the model prediction and experimental data did not exceed 7\%. Hence, a new linear magneto-viscoelastic model was proposed in frequency domain for anisotropic MREs based on López-López et al. model, which predicts the effect of magnetic field on the dynamic shear modulus in function of particle content and frequency.
\end{abstract}

Key words: anisotropic magnetorheological elastomers; fractional derivative model; magnetic field.

\section{Introduction}

Magnetorheological elastomers (MRE) consist of ferromagnetic particles embedded in an elastomeric matrix [1]. When an external magnetic field is applied to these materials their mechanical properties are modified; and they are considered as smart materials.

The particle distribution is an important characteristic of MREs, because their properties are completely dependent on it. Isotropic MRE samples are prepared by vulcanisation without an external magnetic field; these samples have a random particle distribution [2,3]. However, if an external magnetic field is applied during the vulcanisation process, particles are aligned in the direction of the magnetic field, and consequently particle chains or thicker chain aggregates are obtained; these samples are called anisotropic MREs [4,5].

Dynamic properties of anisotropic MREs are dependent on matrix, particle content and magnetic field. The predominant behaviour is the viscoelastic one, due to the nature of the main component of these materials: silicone rubber or a natural rubber [6,7]. That behaviour have been modelled for MRE materials combining different elements as dashpots and springs in different configurations $[8,9]$. In order to obtain a better fitting to experimental data, more elements have been introduced increasing the number of fitting parameters [10]. 
The fractional derivative model can be used to decrease the number of material parameters, and these parameters have a physical meaning [11]. Using these advantages the viscoelastic behaviour of isotropic [7,12] and anisotropic $[13,14]$ MREs have recently been modelled using fractional derivative models. Agirre-Olabide et al [12] have used four material parameters to simulate the viscoelastic behaviour of isotropic MREs and Xu et al. [7] have combined a fractional Kelvin and Maxwell model in parallel configuration developing a higher order model (seven material parameters). Guo et al. [13] have combined an Abel dashpot (fractional derivative element) and a spring in a series configuration, while Zhu et al. [14] have combined in a parallel configuration. Hence, three material parameters have been used to predict the viscoelastic behaviour of anisotropic MREs.

The influence of the magnetic field on the properties of anisotropic MREs has been widely studied. Many models have assumed that perfectly aligned chains were created during the vulcanisation process. Jolly et al. $[15,16]$ have analysed the interaction of two particles using the dipole-dipole moments, while Davis [17] and Shen et al. [18] have studied the interaction of each particle in the whole chain of particles. Bica et al. [4] have used a dipolar magnetic moment approach and the ideal elastic body model.

López-López et al. [19] have proposed a model for magnetorheological fluids introducing the influence of aggregates having a body centered tetragonal (bct) internal structure (a more stable and more realistic structure), and have combined numerical simulations of the composite magnetic permeability with the analytical model predicting the stress-strain relationship. For MREs Leng et al. [20] have proposed an effective permeability model to estimate the shear storage modulus and Dong et al. [21] have developed a theoretical model for chains composed by magnetic particles and a normal pressure, based on the effective permeability calculated by Maxwell Garnett mixing rule. Chen et al. [22] have proposed a finite-column model to simulate the field-induced shear modulus. Quadrate finite columns of particles were simulated to calculate the magnetic permeability of each column and consequently field induced shear modulus. The permeability of isotropic and anisotropic MRE have been estimated using finite element approach [23].

Coupling viscoelastic and magnetic interaction models, few magneto-viscoelastic models have then been developed. A classical four parameter magneto-viscoelastic model have been proposed by Li et al. [24], and all material parameters were fitted to experimental data for each magnetic field densities. The magneto-viscoelastic behaviour using fractional derivatives have been modelled for isotropic [25] and anisotropic [13,14] MREs. Agirre-Olabide et al. [25] have proposed a three-dimensional magneto-viscoelastic model within the linear viscoelastic region for isotropic MREs, coupling a fractional derivative based viscoelastic model with a magnetic field dependant model. On the other hand, the magneto-viscoelastic model proposed in $[13,14]$ for anisotropic MREs have combined in a serial configuration a fractional derivative Maxwell model and a spring, which is dependent on the magnetic field and modelled assuming chain-like structures. The strain amplitudes applied were $0.1 \%$ in [13] and $25 \%$ in [14].

The influence of the magnetic field have been widely modelled for anisotropic MREs, while there are few magneto-viscoelastic models. Moreover, magneto-viscoelastic models used for MREs couples magnetic models assuming a perfect chain-like structures. The aim of this work is to develop a new magneto-viscoelastic model for anisotropic MREs based on both Jolly et al. and López-López et al. models. Thus, we compared these two magnetic field models coupled with a viscoelastic model based on fractional derivatives, with all the fitting parameters having a physical meaning. The main difference between magnetic models is the particle distribution; Jolly et al. [16] model assumes that all particles are assembled into a simple cubic lattice in non-deformed MRE sample, and López-López et al. [19] assumes a bulk column like aggregates in MR samples. A four parameter fractional derivative model was used to predict the viscoelastic behaviour of anisotropic MREs. In order to fit each parameter, five anisotropic MRE samples with different particle volume fractions were synthesised and characterised with a rheometer within the linear viscoelastic (LVE) region, because the modulus variation due to an external magnetic field is larger in that region [26]. The coupling of the viscoelastic and magnetic models was done as in [25]. 


\section{Experimental}

In this work anisotropic magnetorheological elastomers were synthesised using a room temperature vulcanising silicone rubber and soft magnetic particles; five particle contents were analysed. Two different characterisation techniques were performed; dynamic behaviour was measured using a rheometer equipped with a magnetorheological device, and the magnetic properties -with a vibrating sample magnetometer (VSM).

\section{$2.1 \quad$ Preparation of anisotropic samples}

In this study, we have used two components based room temperature vulcanising silicone rubber (RTV-SR); the main matrix WACKER Elastosil® M 4644 A and the vulcaniser WACKER Elastosil ${ }^{\circledR}$ M 4644 B mixed in 10:1 ratio. The embedded soft magnetic spherical particles were carbonyl iron particles HS (BASF The Chemical Company, Germany) with a particle size of $1.25 \pm 0.55 \mu \mathrm{m}$. Samples of five different particle volume fractions were prepared: $0 \%, 5 \%, 10 \%$, $15 \%$ and $20 \%$.

The main matrix (Elastosil@ M $4644 \mathrm{~A}$ ) and particles were mixed at the mentioned contents and when a homogeneous mixture was obtained, the vulcaniser (Elastosil@ M 4644 B) was added. Every time a component was added, vacuum cycles for 30 minutes were applied to remove air bubbles generated during mixing. Finally, the homogenous mixture was poured into a 1-mm-thick mould.

During the vulcanisation process a magnetic field was applied in the thickness direction to obtain a chain alignment of the particles in the direction perpendicular to the shear strain applied during rheometric experiments (figure 1). A magnetic field of a flux density of $130 \mathrm{mT}$ was applied using a pair of permanent magnets places at the both sides of the mould.

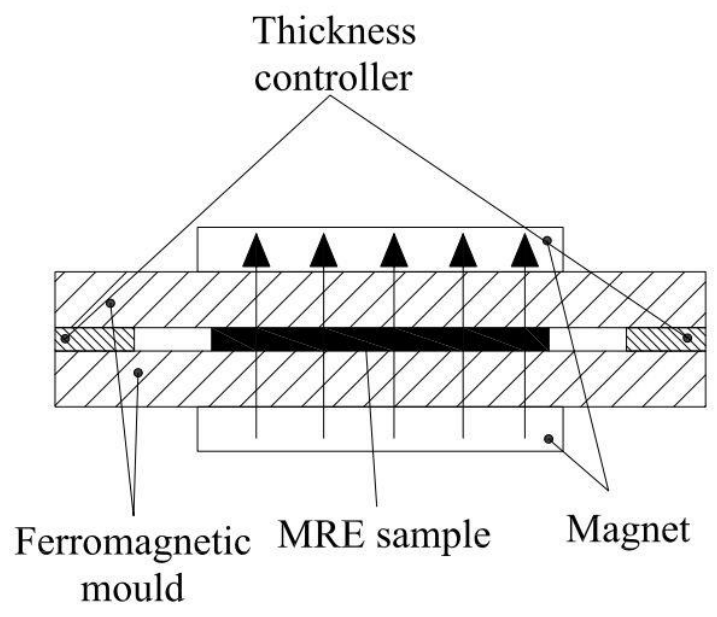

Figure 1. Sketch of the anisotropic MRE sample vulcanising device. 


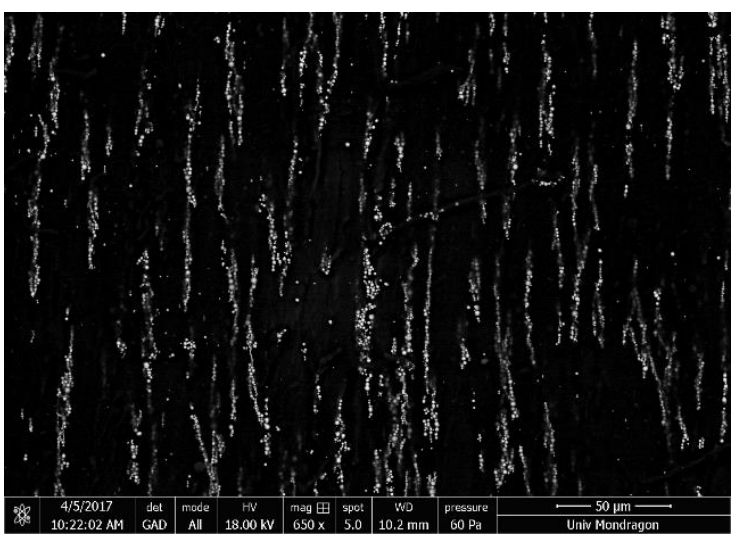

(a)

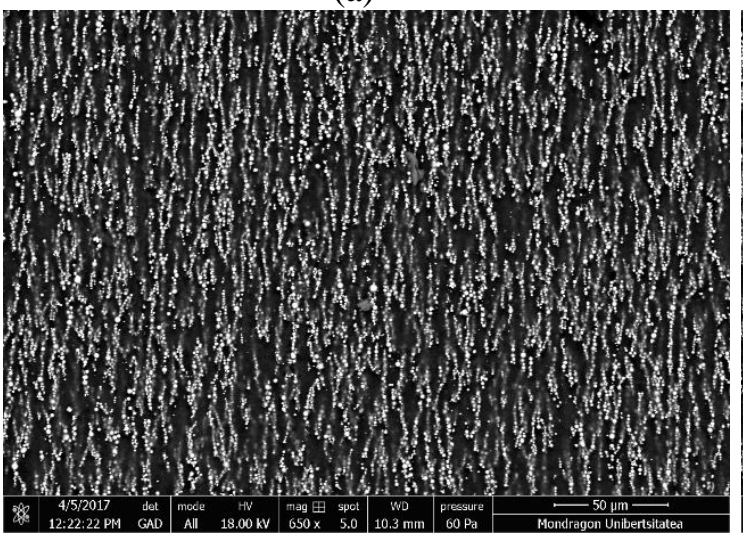

(c)

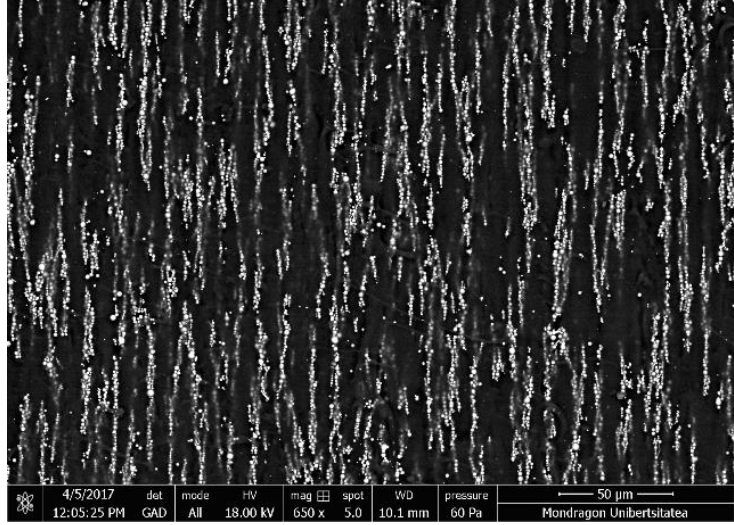

(b)

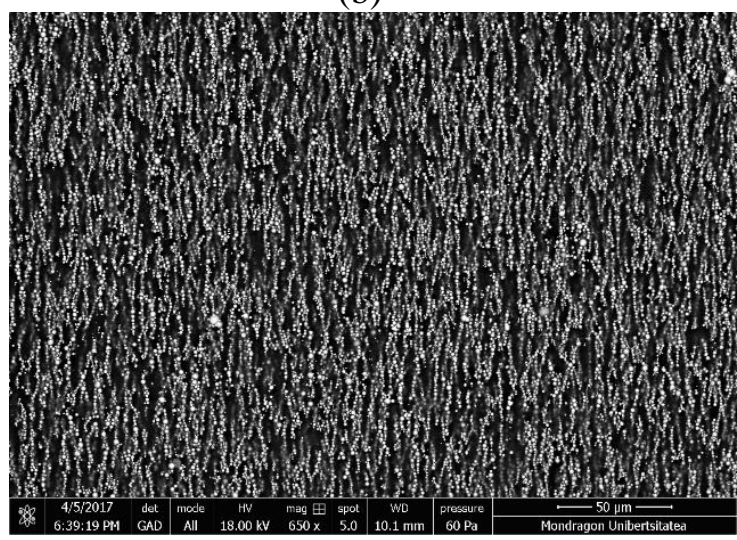

(d)

Figure 2. SEM image of (a) 5\%, (b) 10\%, (c) $15 \%$ and (d) $20 \%$ anisotropic RTV-SR MRE samples in low-vacuum conditions and with a voltage acceleration of $18 \mathrm{kV}$

A Nova Nano SEM 450 scanning electron microscope (SEM) was used to observe the particle alignment and distribution (figure 2 ). The images were taken in a low vacuum condition with an acceleration voltage of $18 \mathrm{kV}$.

\subsection{Magnetorheology}

The dynamic properties of anisotropic MRE were measured using an Anton Paar Physica MCR 501 rheometer equipped with a MRD 70/1T magnetorheological cell, and a parallel plate configuration was used. To avoid the slipping between sample and plates, one of the plates had a serrated surface (PP20/MRD/TI/P2); a normal compressive force of $5 \mathrm{~N}$ was applied to the sample in order to increase the sample contact to the rheometer plates [27]. The sample diameter and thickness was $20 \mathrm{~mm}$ and $1 \mathrm{~mm}$ respectively. To check the reproducibility, three samples were studied for each particle content.

The samples were subjected to torsional deformation generated by a periodic oscillatory rotation of the upper rheometer plate. A strain amplitude of $0.01 \%$ was used in the frequency sweep tests to guarantee that all tests were performed in the linear viscoelastic (LVE) region [28,29]. The frequency range of $0.1-40 \mathrm{~Hz}$ was analysed and divided into two steps; the first one from 0.1 to $10 \mathrm{~Hz}$, and the second one from 10 to $40 \mathrm{~Hz}$. In each step, 30 points were measured, while the acquisition time was fixed to $5 \mathrm{~s}$ per point. The temperature was controlled at $25^{\circ} \mathrm{C}$ using the Julabo F-25 water-based heating/cooling system. Three magnetic field intensities were used: 0 $\mathrm{kA} / \mathrm{m}, 150 \mathrm{kA} / \mathrm{m}$ and $300 \mathrm{kA} / \mathrm{m}$.

\subsection{Vibrating sample magnetometer}

The magnetostatic properties of the anisotropic MRE samples were carried out using a vibrating sample magnetometer (VSM, 4500 EG\&G Princeton Applied Research). This technique consists of a vibrating a sample at a frequency of $85 \mathrm{~Hz}$ in the direction perpendicular to a permanent and nearly homogeneous external magnetic field (figure 3 ). When a magnetic sample is introduced, it is magnetised and consequently it generates its own magnetic field around the sample. Periodic vibrations of the sample produce periodic variation of the magnetic field induced 
by the sample in the laboratory reference frame related to fixed electromagnets. According to the Faraday's induction law, this change produces an electromotive force in pick-up coils, which is measured and directly related to the sample magnetization.

All measurements were performed at room temperature in the field range of $\pm 350 \mathrm{kA} / \mathrm{m}$. The aim of this characterisation was to measure the magnetic susceptibility of the sample in the direction parallel and perpendicular to the particle chains. For measurements of the longitudinal magnetic permeability, a sample with the chains oriented along the electromagnet axis and having diameter of $3 \mathrm{~mm}$ and thickness of $1.08 \pm 0.12 \mathrm{~mm}$ was used (figure 3 (a)). For measurements of the transverse magnetic permeability a sample with the chains oriented perpendicularly to the electromagnet axis and having a rectangular shape with the length of $10.48 \pm 0.82 \mathrm{~mm}$ and thickness of $1 \mathrm{~mm}$ was used (figure 3 (b)). Those samples were held in an $\emptyset 3 \times 20 \mathrm{~mm}$ container. One measurement was performed for each particle content.

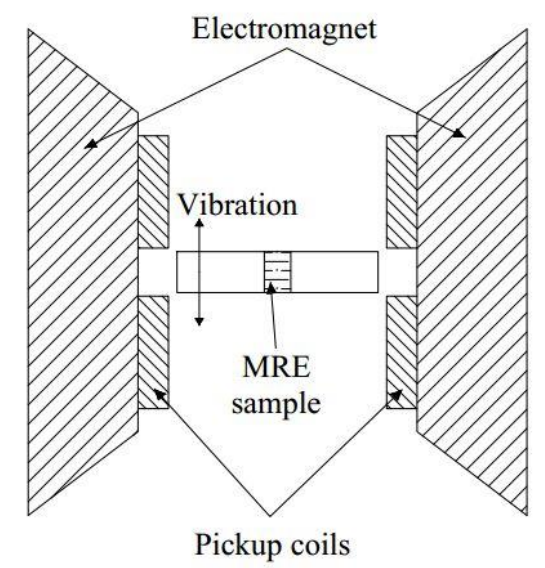

(a)

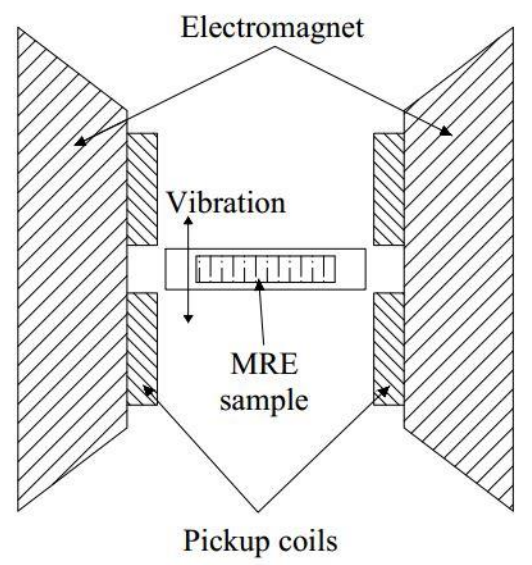

(b)

Figure 3. VSM sketch for particles chains aligned in the (a) parallel and (b) perpendicular direction to the external magnetic field.

From the VSM measurements, the longitudinal and transverse magnetic permeability of each sample was calculated for external fields of 150 and $300 \mathrm{kA} / \mathrm{m}$ - the typical field values used in our rheological experiments.

\section{Anisotropic MRE modelling}

A four parameter fractional derivative model was used and the material parameters were identified using the dynamic properties measured by the rheometer. The influence of the magnetic field was introduced using an empirical magneto-viscoelastic model [25] with the magnetic field contribution to the elastic modulus calculated using both the Jolly et al. [16] model, and the model proposed by López-López et al. [19] for magnetorheological fluids. The last model was combined with experimental magnetic permeability values measured in this work.

\subsection{Fractional derivative model for MRE in the absence of the magnetic field}

The generalised Zener model in the frequency domain written with fractional derivatives contains four parameters,

$$
G^{*}=\frac{G_{0}+\left(G_{0}+C\right)(\mathrm{i} \omega \tau)^{\alpha}}{1+(\mathrm{i} \omega \tau)^{\alpha}}=G_{0}+\frac{C(\mathrm{i} \omega \tau)^{\alpha}}{1+(\mathrm{i} \omega \tau)^{\alpha}},
$$

where $G_{0}$ is the static elastic modulus, $G_{\infty}=G_{0}+C$ is the high frequency limit value of the dynamic modulus, $\tau$ is the relaxation time and $\alpha$ is the fractional parameter [25,11], whose values varies between 0 and 1 .

This model is applied to MRE samples in the absence of field. In figure 4 experimental dynamic properties are shown as a function of frequency and the error does not exceed the 5\%. All samples show the same behaviour, both moduli increase with frequency and particle content. 


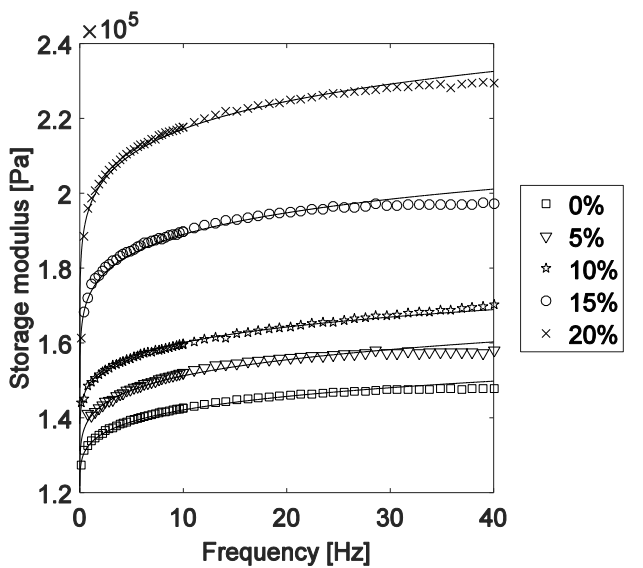

(a)

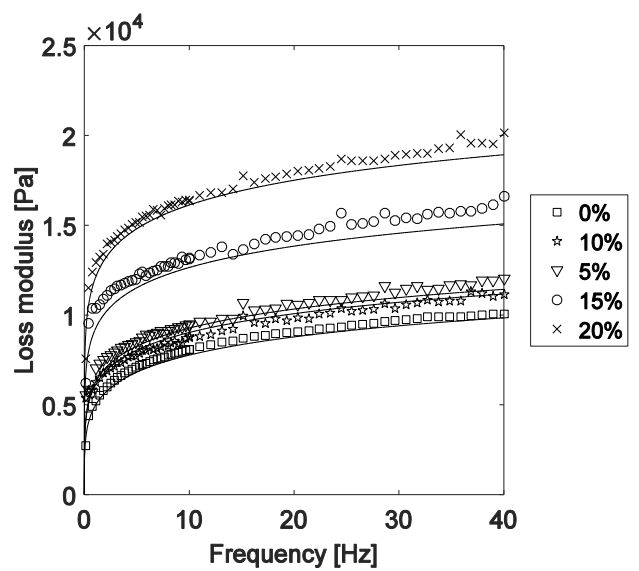

(b)

Figure 4. Dynamic properties of anisotropic MRE samples as a function of frequency, (a) storage and (b) loss modulus. Experimental data are represented as points and the lines correspond to the fitting of equation (1).

After fitting equation (1) to experimental data of each sample (figure 4), the four parameters of the fractional derivative model were obtained (table 1). The fitting was done using the least square method and the minimised error was calculated for the shear complex modulus. The $\mathrm{R}^{2}$ parameter is shown to evaluate the accuracy of the fitting.

Table 1. Fitting parameters of a four parameter fractional derivative model for the anisotropic MRE samples and the

\begin{tabular}{llllll}
\multicolumn{5}{c}{$\mathrm{R} 2$ parameter } \\
\hline & $G_{0}(\mathrm{MPa})$ & $C(\mathrm{MPa})$ & $\tau(\mathrm{s})\left[10^{-7}\right]$ & $\alpha$ & $\mathrm{R}^{2}$ \\
\hline $0 \%$ & 0.120 & 0.157 & 152.1 & 0.253 & 0.997 \\
$5 \%$ & 0.121 & 0.198 & 95.23 & 0.229 & 0.971 \\
$10 \%$ & 0.131 & 0.212 & 51.72 & 0.224 & 0.993 \\
$15 \%$ & 0.138 & 0.379 & 5.919 & 0.180 & 0.965 \\
$20 \%$ & 0.148 & 0.467 & 6.863 & 0.172 & 0.987 \\
\hline
\end{tabular}

In figure 4 the fitting and the experimental results can be seen. The fitting is accurate for all contents in the studied frequency band. However, the fitting is better for the storage modulus than for the loss modulus because the storage modulus is one order of magnitude higher than the loss modulus and consequently its influence on the complex modulus is larger. The larger mean relative error does not exceed the $0.5 \%$ for the storage modulus and $6 \%$ for the loss modulus.

\subsection{Magnetic field effect}

The dynamic properties of anisotropic MREs increase with magnetic field intensity (figure 5), a larger increase was measured with a larger magnetic field.

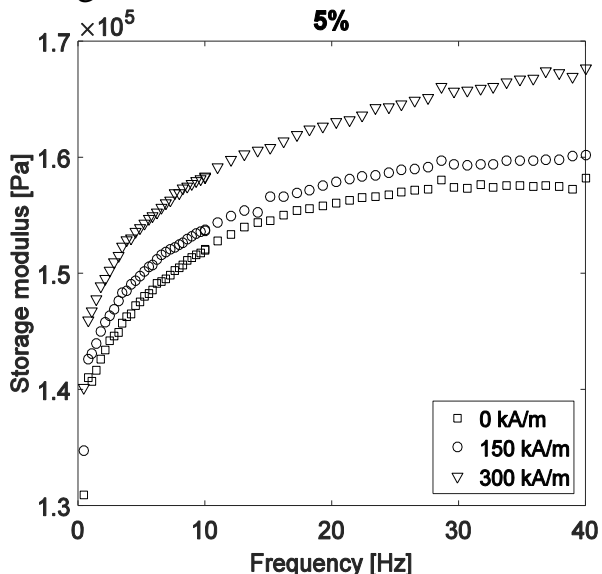

(a)

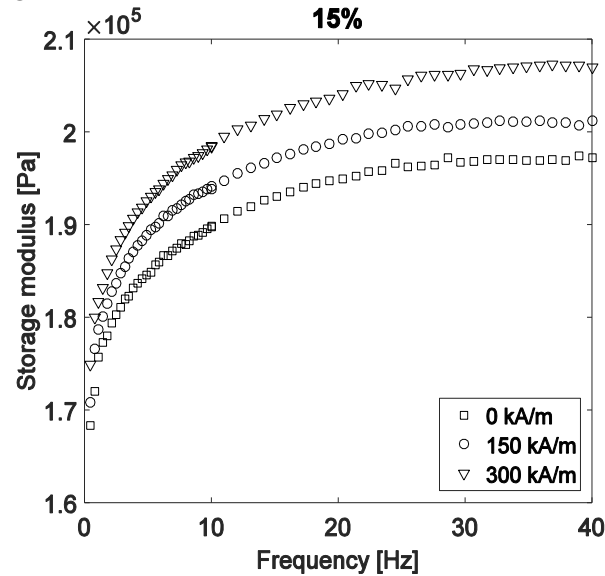

(b)

Figure 5. Influence of the magnetic field on the MRE storage modulus for (a) 5\% and (b) $15 \%$ anisotropic MRE samples as a function of frequency 
In this study, we assumed that the magnetic field only modifies $G_{0}$ and $C$ parameters (equation (1)). Consistency of this assumption has been proved in [25]. These parameters are supposed to depend on magnetic field density $(H)$ and volumetric particle content $(\varphi)$, as follows:

$$
G_{0}(\varphi, H)=G_{0}+\Delta C(\varphi, H) \text { and } C(\varphi, B)=C+\Delta C(\varphi, H) \text {, }
$$

where $G_{0}$ and $C$ corresponds to the fitting values of each particle content (table 1), and $\Delta C$ is elastic modulus variation due to external magnetic field.

The magnetic field dependence was studied using two different models: Jolly et al. model [16] (Sec. 3.1.1) and López-López et al. model [19] (sec. 3.2.2). After determining the influence of the magnetic field, $\Delta C$ was coupled with the fractional derivative model,

$$
G^{*}=\left(G_{0}+\Delta C\right)+\frac{(C+\Delta C)(\mathrm{i} \omega \tau)^{\alpha}}{1+(\mathrm{i} \omega \tau)^{\alpha}} .
$$

\subsubsection{Jolly et al. model}

This model is based on the assumption that all the particles are assembled into a simple cubic lattice in non-deformed MRE sample. When the sample is sheared in the direction perpendicular to the applied magnetic field, the particles are assumed to displace affinely with the elastomer displacement, so the distances between the particles within the chains increase and they experience an attractive magnetic force whose projection to the shear plain produces a macroscopic elastic stress and the elastic modulus is given as follows:

$$
\Delta C=\frac{4 \varphi \mu_{0} M_{\mathrm{p}}^{2} R^{3}}{d^{3}},
$$

where $M_{\mathrm{p}}=3 \beta_{\mathrm{p}} H$ is the induced magnetization of the particle, $\beta_{\mathrm{p}}=\left(\mu_{\mathrm{p}}-1\right) /\left(\mu_{\mathrm{p}}+2\right)$ is the magnetic contrast factor, $\mu_{\mathrm{p}}$ is the particle relative magnetic permeability, $\mu_{0}$ is the vacuum magnetic permeability, $H=H_{0} / \mu$ is the internal magnetic field in the sample, $\mu$ is the relative magnetic permeability of the sample, $R$ is the particle radius, and $d$ is the distance between the centre of two adjacent particles. To determine the distance between particles, equation (5) was used, proposed in [16] for the case of evenly spaced particle chains:

$$
d=2 R \sqrt[3]{\frac{\pi}{6 \varphi}} .
$$

In figure 6 the prediction of the coupling of equations (3) and (4) (lines) and experimental data (points) are shown. At low particle contents the storage modulus is underestimated, while at high particle contents it is overestimated. The discrepancy between the prediction of Jolly model and our experiments varies between 1 and $14 \%$ and increases with the applied field and particle content.

\subsubsection{López-López et al. model}

The López-López et al. model was used for magnetorheological fluids in order to introduce the influence of aggregates generated when an external magnetic field was applied. This model assumes bulk column like aggregates in the MR sample extended along the applied magnetic field - a more stable and more realistic structure observed in experiments for both MR fluids and MRE. The model is based on evaluation of the free energy of the sheared MR sample and the shear stress $\sigma$ is related to the applied strain $\gamma$, as follows:

$$
\begin{aligned}
\sigma= & \mu_{0} H^{2}\left(\mu_{11}-\mu_{\perp}\right) \frac{\gamma}{\left(1+\gamma^{2}\right)^{2}}-\frac{1}{2} \mu_{0} H^{2}\left[\frac{\partial \mu_{11}}{\partial \gamma} \cdot \frac{1}{1+\gamma^{2}}+\frac{\partial \mu_{\perp}}{\partial \gamma} \cdot \frac{\gamma^{2}}{1+\gamma^{2}}\right] \\
& +\frac{1}{2} \mu_{0} H^{2}\left(\mu_{11}-\mu_{\perp}\right) \frac{\gamma}{1+\gamma^{2}}
\end{aligned}
$$


where $H$ is the magnetic field intensity within the samples, $\mu_{\|}$and $\mu_{\perp}$ are the longitudinal and transverse components of the magnetic permeability, the second term containing magnetic permeability derivatives appears to be negligible at strong magnetic fields used in our rheological experiments, so it is neglected. After linearization of equation (6), the following expression was developed,

$$
\Delta C=\frac{3}{2} \mu_{0} H^{2}\left(\mu_{11}-\mu_{\perp}\right),
$$

where, $\Delta C$ is the increment of the zero-frequency storage modulus due to internal magnetic field, $H=H_{0} / \mu_{\|}$, and $H_{0}$ is the applied external magnetic field. From the magnetization measurements (Sec. 2.3), longitudinal and transverse components of the magnetic permeability values were calculated and are shown in table 2; the permeability is larger for high particle content and for lower magnetic fields, and the longitudinal one is larger than the transverse one due to the lower demagnetizing effect in the sample with the aggregates oriented along the applied field.

Table 2. Longitudinal and transverse components of the magnetic permeability of all sample for 150 and $300 \mathrm{kA} / \mathrm{m}$.

\begin{tabular}{lll}
\multicolumn{3}{c}{$150 \mathrm{kA} / \mathrm{m}$} \\
\hline & $\mu_{\|}$ & $\mu_{\perp}$ \\
\hline $5 \%$ & 1.324 & 1.186 \\
$10 \%$ & 1.615 & 1.415 \\
$15 \%$ & 1.950 & 1.512 \\
$20 \%$ & 2.317 & 1.821 \\
\hline
\end{tabular}

\begin{tabular}{llc}
\multicolumn{3}{c}{$300 \mathrm{kA} / \mathrm{m}$} \\
\hline & $\mu_{\|}$ & $\mu_{\perp}$ \\
\hline $5 \%$ & 1.260 & 1.172 \\
$10 \%$ & 1.520 & 1.337 \\
$15 \%$ & 1.683 & 1.500 \\
$20 \%$ & 2.152 & 1.802 \\
\hline
\end{tabular}

Coupling equations (2) and (7), and using experimental magnetic permeability values (table 2 ), the modulus was predicted. In two bottom parts of figure 6 experimental storage modulus is compared to the theoretical one obtained using Eqs. (2) and (7).

The discrepancy between the prediction of López-López model and our experiments varies between 1 and 7\%. It increases with the applied field as in the Jolly model prediction, while there is not a tendency for the particle content. 


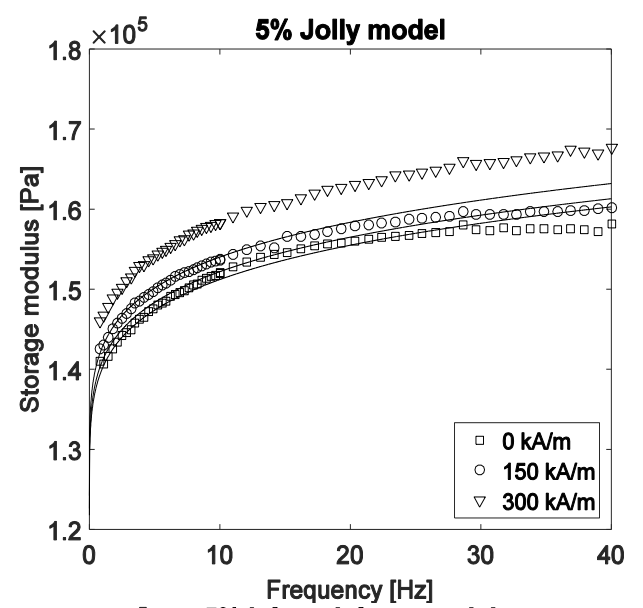

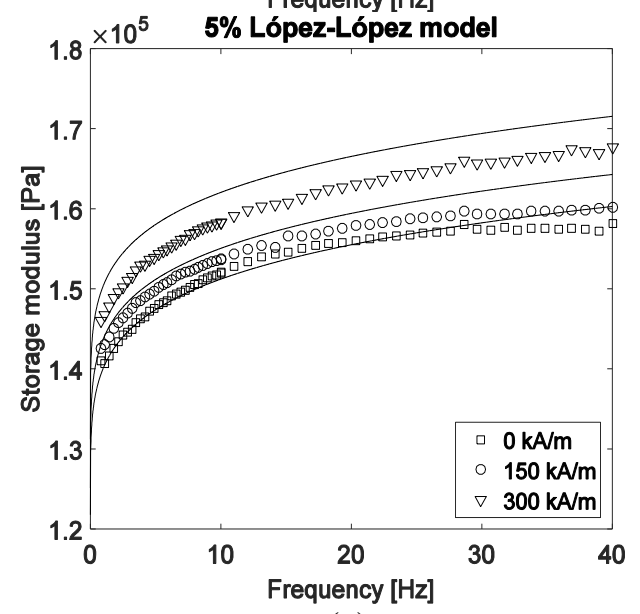

(a)
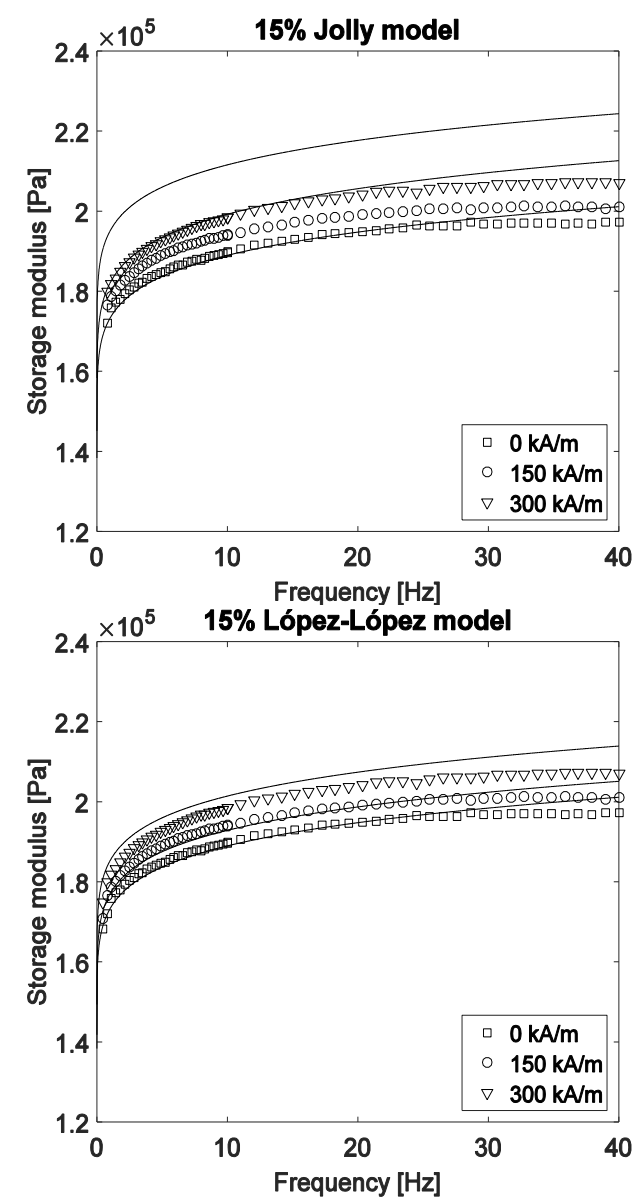

(b)

Figure 6. Influence of magnetic field for (a) $5 \%$ and (b) $15 \%$ anisotropic MRE samples as a function of frequency for the Jolly et al and López-López et al. models.

Comparing both models studied in this work, it can be seen that López-López et al. model seems to be more accurate. The main difference is that for the Jolly et al. model analytical equations were used, while experimental data on the magnetic permeability of each samples were injected into the López-López et al. model.

\section{Conclusions}

In this work a new linear magneto-viscoelastic model was proposed for anisotropic MREs. The four parameter fractional derivative viscoelastic model was successfully fitted to experimental data of anisotropic MREs samples in the absence of the applied field. The mean fitting errors does not exceed the $1 \%$ for the storage modulus and $6 \%$ for the loss modulus.

Two different models allowing calculation of the field-induced part of the storage modulus were coupled with a fractional derivative model. The difference between the magnetic field effect models lies in different microstructures considered. In Jolly et al. model a chain like simple cubic lattice distribution was used, although in López-López et al. model agglomerate bct-chain structures were assumed for MRF. In the López-López et al. model, longitudinal and transverse components of the magnetic permeability of each anisotropic MRE samples were used, which were calculated from magnetometry experimental data. The magnetic permeability is an increasing function of particle content and it decreased with the applied magnetic field.

It was proved that the prediction of the first model was not accurate because the error between the experimental data and the model prediction exceeds $13 \%$ for the storage modulus at the highest magnetic field intensity. Assuming more realistic and mechanically stable bulk aggregates in López-López et al. model, the prediction was more accurate (the error does not exceeds 7\%). Hence a new magneto-viscoelastic model for anisotropic MREs is proposed coupling a four parameter fractional derivative model with the magnetic model of López-López et al., which originally was developed for MRF. 


\section{Acknowledgement}

The authors gratefully acknowledge the financial support from the Department of Education of the Basque Government for the Research Predoc Grant PRE_2014_1_284, PI-2016-1-0026, ACTIMAT and IT009-16 research project; and AVISUINT (DPI 2012-36366) research project from the Spanish Government.

\section{References}

[1] Makarova L A, Alekhina Y A and Perov N S 2016 Peculiarities of magnetic properties of magnetoactive elastomers with hard magnetic filler in crossed magnetic fields $J$. Magn. Magn. Mater. 0-1

[2] Yunus N A, Mazlan S A, Ubaidillah, Choi S, Imaduddin F, Abdul Aziz S A and Ahmad Khairi M H 2016 Rheological properties of isotropic magnetorheological elastomers featuring an epoxidized natural rubber Smart Mater. Struct. 25107001

[3] Gao W and Wang X 2016 Steady shear characteristic and behavior of magneto-thermoelasticity of isotropic MR elastomers Smart Mater. Struct. 2525026

[4] Bica I, Balasoiu M and Kuklin A I 2012 Anisotropic Silicone Rubber Based Magnetorheological Elastomer with Oil Silicone and Iron Microparticles Solid State Phenom. 190 645-8

[5] Aloui S and Klüppel M 2015 Magneto-rheological response of elastomer composites with hybrid-magnetic fillers Smart Mater. Struct. 2425016

[6] Jung H S, Kwon S H, Choi H J, Jung J H and Kim Y G 2016 Magnetic carbonyl iron/natural rubber composite elastomer and its magnetorheology Compos. Struct. 136 $106-12$

[7] Xu Z D, Liao Y X, Ge T and Xu C 2016 Experimental and Theoretical Study of Viscoelastic Dampers with Different Matrix Rubbers J. Eng. Mech. 1424016051

[8] Chen L and Jerrams S 2011 A Rheological Model of the Dynamic Behaviour of Magnetorheological Elastomers J. Appl. Phys. 13513 1-9

[9] Behrooz M, Wang X and Gordaninejad F 2014 Modeling of a new semi-active/passive magnetorheological elastomer isolator Smart Mater. Struct. 2345013

[10] Zhu G, Xiong Y, Daley S and Shenoi R 2015 Magnetorheological elastomer materials and structures with vibration energy control for marine application Analysis and Design of Marine Structures V (CRC Press) pp 197-204

[11] Pritz T 1996 Analysis of four-parameter fractional derivative model of real solid materials J. Sound Vib. 195 103-15

[12] Agirre-Olabide I and Elejabarrieta M J 2016 Maximum attenuation variability of isotropic magnetosensitive elastomers Polym. Test. 54 104-13

[13] Guo F, Du C -b. and Li R -p. 2015 Viscoelastic Parameter Model of Magnetorheological Elastomers Based on Abel Dashpot Adv. Mech. Eng. 6 629386-629386

[14] Zhu J, Xu Z and Guo Y 2013 Experimental and Modeling Study on Magnetorheological Elastomers with Different Matrices J. Mater. Civ. Eng. 25 1762-71

[15] Jolly M R, Carlson J D, C. Muñoz B and Bullions T A 1996 The Magnetoviscoelastic Response of Elastomer Composites Consisting of Ferrous Particles Embedded in a Polymer Matrix J. Intell. Mater. Syst. Struct. 4 613-22

[16] Jolly M R, Carlson J D and Muñoz B C 1999 A model of the behaviour of magnetorheological materials Smart Mater. Struct. 5 607-14

[17] Davis L C 1999 Model of magnetorheological elastomers J. Appl. Phys. 85 3348-51

[18] Shen Y, Golnaraghi M F and Heppler G R 2004 Experimental Research and Modeling of Magnetorheological Elastomers J. Intell. Mater. Syst. Struct. 15 27-35

[19] López-López M T, Kuzhir P, Caballero-Hernández J, Rodríguez-Arco L, Duran J D G and Bossis G 2012 Yield stress in magnetorheological suspensions near the limit of maximum-packing fraction J. Rheol. 561209

[20] Leng D, Sun L, Sun J and Lin Y 2013 Derivation of stiffness matrix in constitutive modeling of magnetorheological elastomer J. Phys. Conf. Ser. 41212028 
[21] Dong X, Ma N, Ou J and Qi M 2013 Predicating magnetorheological effect of magnetorheological elastomers under normal pressure J. Phys. Conf. Ser. 41212035

[22] Chen L, Gong X L and Li W H 2007 Microstructures and viscoelastic properties of anisotropic magnetorheological elastomers Smart Mater. Struct. 16 2645-50

[23] Schubert G and Harrison P 2016 Magnetic induction measurements and identification of the permeability of Magneto-Rheological Elastomers using finite element simulations $J$. Magn. Magn. Mater. 404 205-14

[24] Li W H, Zhou Y and Tian T F 2010 Viscoelastic properties of MR elastomers under harmonic loading Rheol. Acta 49 733-40

[25] Agirre-Olabide I, Lion A and Elejabarrieta M J 2017 A new three-dimensional magnetoviscoelastic model for isotropic magnetorheological elastomers Smart Mater. Struct. 26 35021

[26] Lokander M and Stenberg B 2003 Improving the magnetorheological effect in isotropic magnetorheological rubber materials Polym. Test. 22 677-80

[27] Dong X, Ma N, Qi M, Li J, Chen R and Ou J 2012 The pressure-dependent MR effect of magnetorheological elastomers Smart Mater. Struct. 2175014

[28] Agirre-Olabide I, Berasategui J, Elejabarrieta M J and Bou-Ali M M 2014 Characterization of the linear viscoelastic region of magnetorheological elastomers $J$. Intell. Mater. Syst. Struct. 25 2074-81

[29] Agirre-Olabide I, Elejabarrieta M J and Bou-Ali M M 2015 Matrix dependence of the linear viscoelastic region in magnetorheological elastomers J. Intell. Mater. Syst. Struct. 26 1880-6 